QUATERNIONIC EIGENVALUE PROBLEM

Journal of Mathematical Physics 43, 5815-5829 (2002)

\title{
QUATERNIONIC EIGENVALUE PROBLEM
}

\author{
Stefano De Leo ${ }^{1}$, Giuseppe Scolarici ${ }^{2}$, and Luigi Solombrino ${ }^{2}$ \\ 1 Department of Applied Mathematics, University of Campinas \\ PO Box 6065, SP 13083-970, Campinas, Brazil \\ deleo@ime.unicamp.br \\ 2 Department of Physics, University of Lecce and INFN, Sezione di Lecce \\ PO Box 193, 73100, Lecce, Italy \\ scolarici@le.infn.it \\ solombrino@le.infn.it
}

July, 2002

\begin{abstract}
We discuss the (right) eigenvalue equation for $\mathbb{H}, \mathbb{C}$ and $\mathbb{R}$ linear quaternionic operators. The possibility to introduce an isomorphism between these operators and real/complex matrices allows to translate the quaternionic problem into an equivalent real or complex counterpart. Interesting applications are found in solving differential equations within quaternionic formulations of quantum mechanics.
\end{abstract}

PACS. $02.10 . \mathrm{Tq}-02.10 . \mathrm{Yn}-02.30 . \mathrm{Hq}-02.30 . \mathrm{Tb}-03.65 .-\mathrm{w}$

\section{INTRODUCTION}

The full understanding of the subtleties of the quaternionic eigenvalue problem still represents an intriguing challenge for mathematicians and physicists. The recent study of the eigenvalue problem for complex linear quaternionic operators [1] played a fundamental role in solving quaternionic differential equations [2]. In the last few years, interesting applications of quaternionic analysis and linear algebra were investigated in quantum mechanics [3]. In particular, the solution of the Schrödinger equation in presence of quaternionic perturbations was explicitly given for constant potentials and deviations from standard (complex) quantum mechanics discussed [- $\mid$. In this paper, we aim to complete the study begun in ref. [1] where preliminary steps in solving the eigenvalue problem for complex linear quaternionic operators were traced. In order to extend to the $\mathbb{R}$-linear case the results obtained for the $\mathbb{H}$ and $\mathbb{C}$-linear quaternionic matrices, we have to introduce a system of coupled equations which represents the new eigenvalue problem for $\mathbb{R}$ linear quaternionic operators. It is important to observe that no attempt to develop a complete theory of the quaternionic eigenvalue problem has been made here, this exceeds the scope of our paper. A satisfactory discussion of the eigenvalue problem for quaternionic operators is at present far from being given. We could have directly investigated the eigenvalue equation in the quaternionic space, but we have preferred a more practical approach and chosen to handle the problem by finding a more familiar real or complex space isomorphic to the quaternionic one. We shall show that the isomorphism between $\mathbb{H}, \mathbb{C}$, and $\mathbb{R}$-linear quaternionic operators and real/complex matrices immediately allows to translate the quaternionic (right) eigenvalue problem in a corresponding real or complex counterpart. The study of the new translated problem gives important information about the quaternionic solution. The results obtained are very useful in solving polynomial and differential equations. This could represents a fundamental step in understanding the potentiality of using quaternions in formulating quantum mechanics (by investigating quaternionic deviations from the standard theory [3, (4) and gauge theory (by suggesting new unification groups 印).

Throughout the paper we shall denote by $\mathbb{R}, \mathbb{C}$ and $\mathbb{H}$ the sets of real, complex and quaternionic numbers, $\mathbb{R} \subset \mathbb{C} \subset \mathbb{H}$, and by $V[n, \mathbb{X}]$ and $M[n, \mathbb{X}]$, respectively, the $n$-tuples and the $n \times n$ matrices over $\mathbb{X}$. Linear quaternionic operators will be distinguished by their linearity from the right. In what follows, the notation $\mathcal{O}_{\mathbb{X}}$ stands for quaternionic operators linear (from the right) over the field $\mathbb{X}$. 


\section{QUATERNIONIC ALGEBRA AND LINEAR OPERATORS}

We now introduce the quaternionic algebra and some useful properties of $\mathbb{H}$, $\mathbb{C}$ and $\mathbb{R}$-linear operators. The (real) quaternionic skew-field $\mathbb{H}$ is an associative (division) algebra of rank 4 over $\mathbb{R}$,

$$
q=q_{0}+i q_{1}+j q_{2}+k q_{3}, \quad q_{0,1,2,3} \in \mathbb{R},
$$

where

$$
i^{2}=j^{2}=k^{2}=i j k=-1,
$$

endowed with an involutory anti-automorphism (conjugation)

$$
q \rightarrow \bar{q}=q_{0}-i q_{1}-j q_{2}-k q_{3}
$$

Due to the non-commutative nature of quaternions, we must distinguish between the left and right action of the quaternionic imaginary units $i, j$ and $k$. To do it, we introduce the operators

$$
L_{\mu}=\left(1, L_{i}, L_{j}, L_{k}\right) \quad \text { and } \quad R_{\mu}=\left(1, R_{i}, R_{j}, R_{k}\right), \quad \mu=0,1,2,3,
$$

which act on quaternionic vectors $\psi \in V[n, \mathbb{H}]$ in the following way

$$
L_{\mu} \psi=h_{\mu} \psi \quad \text { and } \quad R_{\mu} \psi=\psi h_{\mu}, \quad h_{\mu}=(1, i, j, k) .
$$

These operators satisfy

$$
L_{i}^{2}=L_{j}^{2}=L_{k}^{2}=L_{i} L_{j} L_{k}=R_{i}^{2}=R_{j}^{2}=R_{k}^{2}=R_{k} R_{j} R_{i}=-1,
$$

and

$$
\left[L_{\mu}, R_{\nu}\right]=0, \quad \mu, \nu=0,1,2,3 .
$$

Note that $\mathbb{H}$-linear quaternionic operators acting on a finite $n$-dimensional quaternionic vector space,

$$
\mathcal{O}_{\mathbb{H}}\left(\psi_{1} q_{1}+\psi_{2} q_{2}\right)=\left(O_{\mathbb{H}} \psi_{1}\right) q_{1}+\left(O_{\mathbb{H}} \psi_{2}\right) q_{2}, \quad q_{1,2} \in \mathbb{H}, \quad \psi_{1,2} \in V[n, \mathbb{H}],
$$

are in one to one correspondence with $n \times n$ quaternionic matrices:

$$
\mathcal{O}_{\mathbb{H}} \leftrightarrow M_{\mathbb{H}} \in M[n, \mathbb{H}]
$$

Consequently, $\mathbb{R}$ and $\mathbb{C}$-linear quaternionic operators [6]

$$
\begin{array}{lll}
\mathcal{O}_{\mathbb{R}}\left(\psi_{1} r_{1}+\psi_{2} r_{2}\right)=\left(\mathcal{O}_{\mathbb{R}} \psi_{1}\right) r_{1}+\left(\mathcal{O}_{\mathbb{R}} \psi_{2}\right) r_{2}, & r_{1,2} \in \mathbb{R}, & \psi_{1,2} \in V[n, \mathbb{H}], \\
\mathcal{O}_{\mathbb{C}}\left(\psi_{1} c_{1}+\psi_{2} c_{2}\right)=\left(\mathcal{O}_{\mathbb{C}} \psi_{1}\right) c_{1}+\left(\mathcal{O}_{\mathbb{C}} \psi_{2}\right) c_{2}, & c_{1,2} \in \mathbb{C}, & \psi_{1,2} \in V[n, \mathbb{H}],
\end{array}
$$

can be represented by $n \times n$ quaternionic matrices $M_{\mathbb{H}}$ and right acting operators $R_{\mu}$ as follows

$$
\mathcal{O}_{\mathbb{R}} \leftrightarrow M_{\mathbb{R}}=\sum_{\mu=0}^{3} M_{\mu, \mathbb{H}} R_{\mu} \quad \text { and } \quad \mathcal{O}_{\mathbb{C}} \leftrightarrow M_{\mathbb{C}}=\sum_{s=0}^{1} M_{s, \mathbb{H}} R_{s} .
$$

Thus, $\mathbb{R}$-linear quaternionic operators consist of right multiplication by quaternionic numbers $(\mu=$ $0,1,2,3)$ whereas $\mathbb{C}$-linear quaternionic operators are restricted to right multiplication by complex numbers $(s=0,1)$.

\section{THE EIGENVALUE PROBLEM}

In this section, we briefly discuss the left and right eigenvalue equation for $\mathbb{H}$, $\mathbb{C}$ and $\mathbb{R}$-linear quaternionic operators. As explicitly shown below, the conceptual difficulties which characterize the left eigenvalue problem readily disappear by resorting to right eigenvalues. The need to apply similarity transformations on $\mathbb{H}, \mathbb{C}$ and $\mathbb{R}$ linear quaternionic matrices introduces complex or real constraints on the right eigenvalues. The choice of complex or real (right) eigenvalues will be extremely useful in finding a practical method of resolution and manipulating quaternionic matrices. 


\section{III-A. LEFT EIGENVALUES}

The left eigenvalue problem for $\mathbb{H}$ linear quaternionic operators reads

$$
\mathcal{O}_{\mathbb{H}} \psi=q \psi, \quad \psi \in V[n, \mathbb{H}], q \in \mathbb{H} .
$$

This problem has been recently studied in the mathematical literature [7, 8]. Nevertheless, no systematic way to approach the problem has been given. We point out some difficulties which appear in solving the left eigenvalue equation.

- Similarity transformations. In finding the solution of Eq. (77), a first difficulty is represented by the impossibility to apply similarity transformations, $S_{\mathbb{H}} \in M[n, \mathbb{H}]$, without losing the formal structure of the left eigenvalue equation. In fact, by observing that $S_{\mathbb{H}} q \neq q S_{\mathbb{H}}$, the quaternionic matrices

$$
M_{\mathbb{H}} \quad \text { and } \quad S_{\mathbb{H}} M_{\mathbb{H}} S_{\mathbb{H}}^{-1}
$$

do not necessarily satisfy the same eigenvalue equation. Consequently, we can have quaternionic matrices with the same left eigenvalue spectrum, but no similarity transformation relating them. Explicit example are found in ref. [1].

- Hermitian operators. Let $\mathcal{O}_{\mathbb{H}}$ be an hermitian quaternionic operator, $\psi$ the eigenvector corresponding to the eigenvalue $q$. By using Eq. (7) and denoting by $\langle\varphi \mid \psi\rangle$ the inner product in $V[n, \mathbb{H}]$, we obtain

$$
0=\left\langle\mathcal{O}_{\mathbb{H}} \psi \mid \psi\right\rangle-\left\langle\psi \mid \mathcal{O}_{\mathbb{H}} \psi\right\rangle \Rightarrow 0=\langle q \psi \mid \psi\rangle-\langle\psi \mid q \psi\rangle \neq(\bar{q}-q)\langle\psi \mid \psi\rangle .
$$

Consequently, the left eigenvalue problem for hermitian operators could admit quaternionic solutions [9].

- Square operators and eigenvalues. As a last difficulty in the use of left eigenvalues, we observe that if $\psi$ is an $\mathcal{O}_{\mathbb{H}}$ eigenvector with eigenvalue $q$, it will not necessarily be an $\mathcal{O}_{\mathbb{H}}^{2}$ eigenvector with eigenvalue $q^{2}$. In fact,

$$
\mathcal{O}_{\mathbb{H}}^{2} \psi=\mathcal{O}_{\mathbb{H}} q \psi \neq q \mathcal{O}_{\mathbb{H}} \psi=q^{2} \psi \text {. }
$$

\section{III-B. RIGHT EIGENVALUES}

The right eigenvalue equation for $\mathbb{H}$-linear transformations reads

$$
\mathcal{O}_{\mathbb{H}} \psi=\psi q, \quad \psi \in V[n, \mathbb{H}], q \in \mathbb{H} .
$$

Such an equation can be reduced to a right complex eigenvalue equation rephasing the quaternionic eigenvalues by unitary quaternions $u$,

$$
\mathcal{O}_{\mathbb{H}} \psi u=\psi u \bar{u} q u=\psi u z, \quad z \in \mathbb{C} .
$$

This trick obviously fails for complex and real linear transformations. In fact, due to the presence of the operators $R_{i}$ in $\mathcal{O}_{\mathbb{C}}$ and $\boldsymbol{R} \equiv\left(R_{i}, R_{j}, R_{k}\right)$ in $\mathcal{O}_{\mathbb{R}}$, we cannot apply unitary transformations from the right. Observe that

$$
\left(\mathcal{O}_{\mathbb{R}, \mathrm{c}} \psi\right) u \neq \mathcal{O}_{\mathbb{R}, \mathbb{C}}(\psi u), \quad u \in \mathbb{H} .
$$

The failure of the associativity in Eq. (11) suggests that we should consider complex eigenvalue equations for $\mathbb{C}$-linear quaternionic operators,

$$
\mathcal{O}_{\mathbb{C}} \psi=\psi z, \quad z \in \mathbb{C},
$$

and real eigenvalues for $\mathbb{R}$-linear quaternionic operators,

$$
\mathcal{O}_{\mathbb{R}} \psi=\psi r, \quad r \in \mathbb{R} .
$$

These equations are formally invariant under $\mathbb{C}$ and $\mathbb{R}$-linear similarity transformations. Moreover, it can easily be proved that

$$
\mathcal{O}_{\mathbb{H}}^{n} \psi=\psi q^{n}, \quad \mathcal{O}_{\mathrm{C}}^{n} \psi=\psi z^{n}, \quad \mathcal{O}_{\mathbb{R}}^{n} \psi=\psi r^{n} .
$$

It is important to note here that $\mathbb{R}$-linear quaternionic operators admit real eigenvalues only in particular cases. Thus, Eq.(13) has to be generalized. As shown later, a satisfactory discussion of the eigenvalue problem for $\mathbb{R}$-linear quaternionic operators will require the use of a system of coupled equations. 


\section{CANONICAL FORMS}

In this section, following the procedure introduced in the paper of ref. [1], we discuss the canonical forms for $\mathbb{H}, \mathbb{C}$ and $\mathbb{R}$-linear quaternionic matrices. The results we will establish find an immediate application in the theory of quaternionic differential operators. In fact, by using the canonical form $J_{\mathbb{X}}$ of a given matrix $M_{\mathbb{X}}$ we can readily obtain the exponential

$$
\exp \left[M_{\mathbb{X}} x\right]=S_{\mathbb{X}} \exp \left[J_{\mathbb{X}} x\right] S_{\mathbb{X}}^{-1}
$$

and consequently, avoiding tedious calculations, to solve quaternionic differential equations with constant coefficients [2].

\section{IV-A. HI-LINEAR MATRICES}

While matrices over commutative rings have gained much attention, the literature on matrices with quaternionic entries is often fragmentary. The main difficulty is that, due to the noncommutative nature of quaternions, the standard method of resolution breaks down. Consequently, finding eigenvalues and canonical forms represents a more delicate problem. The recent renewed interest in quaternionic matrix theory [1, 10] and its applications [2] shed new light on this intriguing research field. To facilitate access to the individual topics, we recall the main properties of $\mathbb{H}$-linear quaternionic matrices 11, 12, 13, 14 and repeat the relevant theorems from [1], 10, 15, 16] without proofs, thus making our exposition self-contained.

In approaching the problem of diagonalization we have to consider a right quaternionic eigenvalue equation. In fact, from

$$
M_{\mathbb{H}} \psi_{k}=\psi_{k} q_{k}, \quad k=1, \ldots, n, \quad \psi_{k} \in V[n, \mathbb{H}],
$$

in the case $M_{\mathbb{H}}$ is diagonalizable, we immediately get the following matrix equation

$$
M_{\mathbb{H}} S_{\mathbb{H}}\left[\psi_{1}, \psi_{2}, \ldots, \psi_{n}\right]=S_{\mathbb{H}}\left[\psi_{1}, \psi_{2}, \ldots, \psi_{n}\right] D_{\mathbb{H}},
$$

where

$$
D_{\mathbb{H}}=\operatorname{diag}\left[q_{1}, q_{2}, \ldots, q_{n}\right]
$$

and $S_{\mathbb{H}}=S_{\mathbb{H}}\left[\psi_{1}, \psi_{2}, \ldots, \psi_{n}\right]$ is defined by $\operatorname{Col}_{k}\left(S_{\mathbb{H}}\right)=\psi_{k}$.

Consequently, the diagonalization of the matrix $M_{\mathbb{H}}$

$$
M_{\mathbb{H}}=S_{\mathbb{H}} D_{\mathbb{H}} S_{\mathbb{H}}^{-1}
$$

is obtained by solving the corresponding right eigenvalue problem. It is important to note here that we have infinite ways to diagonalize a quaternionic matrix $M_{\mathbb{H}}$,

$$
\operatorname{diag}\left[u_{1}, u_{2}, \ldots, u_{n}\right] \operatorname{diag}\left[q_{1}, q_{2}, \ldots, q_{n}\right] \operatorname{diag}\left[\bar{u}_{1}, \bar{u}_{2}, \ldots, \bar{u}_{n}\right]
$$

Geometrically speaking this means that $\operatorname{Im}[q]$ can arbitrarily be fixed on the sphere of $\operatorname{ray}|\operatorname{Im}[q]|$. By a particular choice of the unitary matrix

$$
U_{\mathbb{H}}=\operatorname{diag}\left[u_{1}, u_{2}, \ldots, u_{n}\right]
$$

we can set a preferred space direction, for example the positive $i$ axis, and consequently a complex (positive) eigenvalue spectrum.

Let us now briefly recall some properties of the eigenvalue spectrum of $\mathbb{H}$ linear quaternionic matrices. By using the symplectic decomposition of the matrix $M_{\mathbb{H}}$

$$
M_{\mathbb{H}}=M_{1}+j M_{2}, \quad M_{1,2} \in M[n, \mathbb{C}],
$$

and the symplectic decomposition of the vector $\psi$,

$$
\psi=\psi_{1}+j \psi_{2}, \quad \psi_{1,2} \in V[n, \mathbb{C}],
$$


we can rewrite Eq. 10 ) in the following (complex) form

$$
\tilde{M}_{\mathbb{H}} \tilde{\psi}=z \tilde{\psi},
$$

where

$$
\tilde{M}_{\mathbb{H}}=\left(\begin{array}{cr}
M_{1} & -M_{2}^{*} \\
M_{2} & M_{1}^{*}
\end{array}\right) \in M[2 n, \mathbb{C}] \quad \text { and } \quad \tilde{\psi}=\left(\begin{array}{l}
\psi_{1} \\
\psi_{2}
\end{array}\right) \in V[2 n, \mathbb{C}]
$$

The mapping

$$
f: M_{\mathbb{H}} \longmapsto \tilde{M}_{\mathbb{H}}
$$

is an isomorphism of the ring of quaternionic matrices $M_{\mathbb{H}}$ into the ring of the corresponding complex counterparts $\tilde{M}_{\mathbb{H}}$. It is important to observe that this isomorphism do not preserve the inner product of eigenvectors [9]. Nevertheless, the choice of a complex projection of quaternionic inner products [17] opens the door to interesting applications in relativistic quantum mechanics [18]. The complex orthogonality of quaternionic eigenvectors (instead of a quaternionic orthogonality) implies a doubling of solution in the two-dimensional quaternionic Dirac equation 17. The four (complex) orthogonal quaternionic solutions describe particle/antiparticle with spin up/down. The use of complex inner products is also a fundamental ingredient in the formulation of gauge theories by geometric algebras [5].

The next theorem states the main property of the eigenvalue spectrum of $\mathbb{H}$ linear quaternionic matrices, for a detailed discussion see refs. [14, 15, 16].

\section{Theorem 1.}

Let $\tilde{M}_{\mathbb{H}}$ be the matrix given in Eq.(10). Then, its eigenvalues appear in complex conjugate pairs.

By using the result of Theorem 1 and the Gram-Schmidt method, we can readily obtain the triangular form for $\mathbb{H}$-linear quaternionic matrices.

\section{Theorem 2.}

Every $M_{\mathbb{H}}$ is unitarily similar to an upper triangular matrix.

Moreover, a Jordan form can be given for every $\mathbb{H}$-linear quaternionic matrix.

\section{Theorem 3.}

Every $n \times n$ matrix with real quaternion elements is similar under a matrix transformation with real quaternion elements to a matrix in (complex) Jordan normal form with diagonal elements in the complex field.

To prove Theorem 3 , we can use the isomorphism defined in (17). To any $\tilde{M}_{\mathbb{H}}$ corresponds a $2 n \times 2 n$ matrix $B$ in the (complex) Jordan form $B=B_{\mathbb{H}} \oplus B_{\mathbb{H}}^{*}$, such that $\tilde{M}_{\mathbb{H}} P=P B$, where the (non-singular) matrix $P$ has the form

$$
P=\left(\begin{array}{cc}
P_{1} & -P_{2}^{*} \\
P_{2} & P_{1}^{*}
\end{array}\right)
$$

This implies that

$$
\left(P_{1}+j P_{2}\right)^{-1} M_{\mathbb{H}}\left(P_{1}+j P_{2}\right)=B_{\mathbb{H}}=D_{\mathbb{H}}+N_{\mathbb{H}},
$$

where $D_{\mathbb{H}}$ and $N_{\mathbb{H}}$ respectively denote the diagonal and the nilpotent parts of $B_{\mathbb{H}}$.

\section{IV-B. C-LINEAR MATRICES}

Let us now consider $\mathbb{C}$-linear transformations. We can associate to any $n \times n \mathbb{C}$-linear quaternionic matrix a $2 n$-dimensional complex matrix by the following mapping

$$
M_{\mathbb{C}}=M_{\mathbb{H}}+M_{\mathbb{H}}^{\prime} R_{i} \longleftrightarrow \tilde{M}_{\mathbb{C}}=f\left(M_{\mathbb{H}}\right)+i f\left(M_{\mathbb{H}}^{\prime}\right)=\left(\begin{array}{cr}
M_{1} & -M_{2}^{*} \\
M_{2} & M_{1}^{*}
\end{array}\right)+i\left(\begin{array}{cc}
M_{1}^{\prime} & -M_{2}^{\prime *} \\
M_{2}^{\prime} & M_{1}^{\prime *}
\end{array}\right),
$$

where $f$ denotes the isomorphism defined in (17). Then the following proposition holds: 


\section{Proposition 1.}

Let $M_{\mathbb{C}}$ be a $\mathbb{C}$-linear quaternionic matrix and $\tilde{M}_{\mathbb{C}}$ its complex counterpart (see Eq. (19)). The mapping

$$
g: M_{\mathbb{C}} \longmapsto \tilde{M}_{\mathbb{C}}
$$

is an isomorphism of the ring of the $n$-dimensional $\mathbb{C}$-linear matrices into the ring of $2 n$-dimensional complex matrices.

Indeed, if

$$
A_{\mathbb{C}}=A_{0, \mathrm{HH}}+A_{1, \mathbb{H}} R_{i} \text { and } B_{\mathbb{C}}=B_{0, \mathrm{H}}+B_{1, \mathrm{H}} R_{i}
$$

are two $\mathbb{C}$-linear matrices, their corresponding complex counterparts are given by

$$
g\left(A_{\mathbb{C}}\right)=f\left(A_{0, \mathbb{H}}\right)+i f\left(A_{1, \mathbb{H}}\right), \quad g\left(B_{\mathbb{C}}\right)=f\left(B_{0, \mathbb{H}}\right)+i f\left(B_{1, \mathbb{H}}\right)
$$

Then,

$$
\begin{aligned}
A_{\mathbb{C}} B_{\mathbb{C}} & =C_{\mathbb{C}} \\
& =A_{0, \mathbb{H}} B_{0, \mathbb{H}}+A_{1, \mathbb{H}} R_{i} B_{0, \mathbb{H}}+A_{0, \mathbb{H}} B_{1, \mathbb{H}} R_{i}+A_{1, \mathbb{H}} R_{i} B_{1, \mathbb{H}} R_{i} \\
& =A_{0, \mathbb{H}} B_{0, \mathbb{H}}-A_{1, \mathbb{H}} B_{1, \mathbb{H}}+\left(A_{0, \mathbb{H}} B_{1, \mathbb{H}}+A_{1, \mathbb{H}} B_{0, \mathbb{H}}\right) R_{i}
\end{aligned}
$$

and

$$
\begin{aligned}
g\left(A_{\mathbb{C}}\right) g\left(B_{\mathbb{C}}\right) & =f\left(A_{0, \mathbb{H}}\right) f\left(B_{0, \mathbb{H}}\right)-f\left(A_{1, \mathbb{H}}\right) f\left(B_{1, \mathbb{H}}\right)+i f\left(A_{1, \mathbb{H}}\right) f\left(B_{0, \mathbb{H}}\right)+i f\left(A_{0, \mathbb{H}}\right) f\left(B_{1, \mathbb{H}}\right) \\
& =f\left(A_{0, \mathbb{H}} B_{0, \mathbb{H}}-A_{1, \mathbb{H}} B_{1, \mathbb{H}}\right)+i f\left(A_{0, \mathbb{H}} B_{1, \mathbb{H}}+A_{1, \mathbb{H}} B_{0, \mathbb{H}}\right) \\
& =g\left(C_{\mathbb{C}}\right) \square
\end{aligned}
$$

By using this isomorphism, the right eigenvalue spectrum of $\mathbb{C}$-linear quaternionic matrices can easily be determined [1]. The following result

"A $\mathbb{C}$-linear matrix is diagonalizable if the corresponding complex counterpart is diagonalizable".

was proven in Ref. [1] (where a preliminary discussion of the eigenvalue problem for $\mathbb{C}$-linear quaternionic matrix operators was given). It is worth pointing out that the converse of the previous statement is, in general, not true. For instance, let us consider the complex matrix

$$
\tilde{G}_{\mathbb{C}}=\left(\begin{array}{cccc}
z_{1} & 0 & 1 & 0 \\
0 & z_{2} & 0 & 1 \\
0 & 0 & z_{1} & 0 \\
0 & 0 & 0 & z_{2}
\end{array}\right) .
$$

This matrix admits a corresponding diagonalizable $\mathbb{C}$-linear quaternionic matrix given by

$$
G_{\mathbb{C}}=D_{\mathbb{C}}+N_{\mathbb{C}}=\left(\begin{array}{cc}
\operatorname{Re}\left(z_{1}\right)+\operatorname{Im}\left(z_{1}\right) R_{i} & 0 \\
0 & \operatorname{Re}\left(z_{2}\right)+\operatorname{Im}\left(z_{2}\right) R_{i}
\end{array}\right)+\frac{1}{2}\left(\begin{array}{cc}
-j+k R_{i} & 0 \\
0 & -j+k R_{i}
\end{array}\right),
$$

where $N_{\mathbb{C}}$ is nilpotent, diagonal and commutes with $D_{\mathbb{C}}$.

The normal form of a $\mathbb{C}$-linear quaternionic matrix can easily be calculated. Indeed, given any $\mathbb{C}$-linear transformation $M_{\mathbb{C}}$ and its corresponding complex counterpart $\tilde{M}_{\mathbb{C}}$ [see Eq.(19)], from the known properties of the Jordan form of complex matrices, we can immediately obtain

$$
\tilde{S}_{\mathbb{C}}^{-1} \tilde{M}_{\mathbb{C}} \tilde{S}_{\mathbb{C}}=\tilde{J}_{\mathbb{C}}=\tilde{D}_{\mathbb{C}}+\tilde{N}_{\mathbb{C}},
$$

where $\tilde{D}_{\mathbb{C}}$ is diagonal, $\tilde{N}_{\mathbb{C}}$ is nilpotent and $\left[\tilde{D}_{\mathbb{C}}, \tilde{N}_{\mathbb{C}}\right]=0$. Then, the quaternionic $\mathbb{C}$-linear matrices $M_{\mathbb{C}}, S_{\mathbb{C}}, D_{\mathbb{C}}$, and $N_{\mathbb{C}}$ are uniquely determined by the isomorphism stated in Proposition 1.

\section{IV-C. $\mathbb{R}$-LINEAR MATRICES}

In the $n$ dimensional quaternionic vector space $V[n, \mathbb{H}]$, the $\mathbb{R}$-linear transformations are represented by

$$
M_{\mathbb{R}}=\sum_{\mu=0}^{3} M_{\mu, \mathbb{H}} R_{\mu},
$$


where $M_{\mu, \mathrm{H}}$ represent $\mathbb{H}$ linear quaternionic matrices and $R_{\mu}$ are the right acting operators defined in the second section. Any $M_{\mathbb{R}}$ is then characterized by $16 n^{2}$ real parameters. We can translate $\mathbb{R}$-linear $n \times n$ matrices into equivalent $4 n \times 4 n$ real matrices, and vice-versa, by the following translation rules:

$$
\begin{aligned}
& R_{i} \longleftrightarrow \mathbf{I}=\left(\begin{array}{cccc}
0 & -\mathbf{1}_{n} & 0 & 0 \\
\mathbf{1}_{n} & 0 & 0 & 0 \\
0 & 0 & 0 & \mathbf{1}_{n} \\
0 & 0 & -\mathbf{1}_{n} & 0
\end{array}\right) \\
& R_{j} \longleftrightarrow \mathbf{J}=\left(\begin{array}{cccc}
0 & 0 & -\mathbf{1}_{n} & 0 \\
0 & 0 & 0 & -\mathbf{1}_{n} \\
\mathbf{1}_{n} & 0 & 0 & 0 \\
0 & \mathbf{1}_{n} & 0 & 0
\end{array}\right), \\
& R_{k} \longleftrightarrow \mathbf{K}=\left(\begin{array}{cccc}
0 & 0 & 0 & -\mathbf{1}_{n} \\
0 & 0 & \mathbf{1}_{n} & 0 \\
0 & -\mathbf{1}_{n} & 0 & 0 \\
\mathbf{1}_{n} & 0 & 0 & 0
\end{array}\right)
\end{aligned}
$$

and

$$
M_{\mu, \mathrm{H}}=M_{0}+i M_{1}+j M_{2}+k M_{3} \longleftrightarrow \hat{M}_{\mu, \mathrm{H}}=\left(\begin{array}{rrrr}
M_{0} & -M_{1} & -M_{2} & -M_{3} \\
M_{1} & M_{0} & -M_{3} & M_{2} \\
M_{2} & M_{3} & M_{0} & -M_{1} \\
M_{3} & -M_{2} & M_{1} & M_{0}
\end{array}\right)
$$

where $M_{\mu, \mathbb{H}} \in M[n, \mathbb{H}], M_{0, \ldots, 3} \in M[n, \mathbb{R}]$, and $\hat{M}_{\mu, \mathbb{H}} \in M[4 n, \mathbb{R}]$. It is easy to verify that $\mathbf{I}, \mathbf{J}, \mathbf{K}$ commute with $\hat{M}_{\mu, \text { H }}$

$$
\left[\mathbf{I}, \hat{M}_{\mu, \mathrm{H}}\right]=\left[\mathbf{J}, \hat{M}_{\mu, \mathrm{H}}\right]=\left[\mathbf{K}, \hat{M}_{\mu, \mathrm{H}}\right]=0
$$

and

$$
\mathbf{I}^{2}=\mathbf{J}^{2}=\mathbf{K}^{2}=\mathbf{K J I}=-\mathbf{1}
$$

The following proposition holds.

\section{Proposition 2.}

Let $M_{\mathbb{R}}$ be a $\mathbb{R}$-linear matrix and $\hat{M}_{\mathbb{R}}$ its real counterpart; then the mapping

$$
h: M_{\mathbb{R}}=M_{0, \mathbb{H}}+M_{1, \mathbb{H}} R_{i}+M_{2, \mathbb{H}} R_{j}+M_{3, \mathbb{H}} R_{k} \longmapsto \hat{M}_{\mathbb{R}}=\hat{M}_{0, \mathbb{H}}+\hat{M}_{1, \mathbb{H}} \mathbf{I}+\hat{M}_{2, \mathbb{H}} \mathbf{J}+\hat{M}_{3, \mathbb{H}} \mathbf{K}
$$

is an isomorphism of the ring of the $n$-dimensional $\mathbb{R}$-linear matrices into the ring of $4 n$-dimensional real matrices $\hat{M}_{\mathbb{R}}$.

Observe that $\widehat{M_{\mathbb{H}} M_{\mathbb{H}}^{\prime}}=\hat{M}_{\mathbb{H}} \hat{M}_{\mathbb{H}}^{\prime}$. Let

$$
A_{\mathbb{R}}=A_{0, \mathrm{HH}}+A_{1, \mathrm{H}} R_{i}+A_{2, \mathrm{H}} R_{j}+A_{3, \mathrm{HH}} R_{k} \quad \text { and } \quad B_{\mathbb{R}}=B_{0, \mathrm{HH}}+B_{1, \mathrm{HH}} R_{i}+B_{2, \mathrm{HH}} R_{j}+B_{3, \mathrm{HH}} R_{k}
$$

be two $\mathbb{R}$-linear quaternionic matrices. Their corresponding real counterparts are given by

$$
h\left(A_{\mathbb{R}}\right)=\hat{A}_{0, \mathbb{H}}+\mathbf{I} \hat{A}_{1, \mathbb{H}}+\mathbf{J} \hat{A}_{2, \mathbb{H}}+\mathbf{K} \hat{A}_{3, \mathbb{H}} \quad \text { and } \quad h\left(B_{\mathbb{R}}\right)=\hat{B}_{0, \mathbb{H}}+\mathbf{I} \hat{B}_{1, \mathbb{H}}+\mathbf{J} \hat{B}_{2, \mathbb{H}}+\mathbf{K} \hat{B}_{3, \mathbb{H}}
$$

Then,

$$
\begin{aligned}
& A_{\mathbb{R}} B_{\mathbb{R}}=C_{\mathbb{R}} \\
& =\left(A_{0, \mathbb{H}} B_{0, \mathbb{H}}-A_{1, \mathbb{H}} B_{1, \mathbb{H}}-A_{2, \mathbb{H}} B_{2, \mathbb{H}}-A_{3, \mathbb{H}} B_{3, \mathbb{H}}\right)+ \\
& \left(A_{0, \mathbb{H}} B_{1, \mathbb{H}}+A_{1, \mathbb{H}} B_{0, \mathbb{H}}+A_{2, \mathbb{H}} B_{3, \mathbb{H}}-A_{3, \mathbb{H}} B_{2, \mathrm{H}}\right) R_{i}+ \\
& \left(A_{0, \mathbb{H}} B_{2, \mathbb{H}}+A_{2, \mathbb{H}} B_{0, \mathbb{H}}+A_{3, \mathbb{H}} B_{1, \mathbb{H}}-A_{1, \mathbb{H}} B_{3, \mathbb{H}}\right) R_{j}+ \\
& \left(A_{0, \mathbb{H}} B_{3, \mathbb{H}}+A_{3, \mathbb{H}} B_{0, \mathbb{H}}+A_{1, \mathbb{H}} B_{2, \mathbb{H}}-A_{2, \mathrm{H}} B_{1, \mathbb{H}}\right) R_{k}
\end{aligned}
$$


and

$$
\begin{aligned}
h\left(A_{\mathbb{R}}\right) h\left(B_{\mathbb{R}}\right)= & \left(\hat{A}_{0, \mathrm{H}} \hat{B}_{0, \mathrm{H}}-\hat{A}_{1, \mathrm{H}} \hat{B}_{1, \mathrm{H}}-\hat{A}_{2, \mathrm{HH}} \hat{B}_{2, \mathrm{H}}-\hat{A}_{3, \mathrm{H}} \hat{B}_{3, \mathrm{H}}\right)+ \\
& \left(\hat{A}_{0, \mathrm{H}} \hat{B}_{1, \mathrm{H}}+\hat{A}_{1, \mathrm{H}} \hat{B}_{0, \mathrm{H}}+\hat{A}_{2, \mathrm{H}} \hat{B}_{3, \mathrm{H}}-\hat{A}_{3, \mathrm{H}} \hat{B}_{2, \mathrm{H}}\right) \mathbf{I}+ \\
& \left(\hat{A}_{0, \mathrm{HH}} \hat{B}_{2, \mathrm{H}}+\hat{A}_{2, \mathrm{H}} \hat{B}_{0, \mathrm{H}}+\hat{A}_{3, \mathrm{H}} \hat{B}_{1, \mathrm{H}}-\hat{A}_{1, \mathrm{H}} \hat{B}_{3, \mathrm{H}}\right) \mathbf{J}+ \\
& \left(\hat{A}_{0, \mathrm{H}} \hat{B}_{3, \mathrm{H}}+\hat{A}_{3, \mathrm{H}} \hat{B}_{0, \mathrm{H}}+\hat{A}_{1, \mathrm{H}} \hat{B}_{2, \mathrm{H}}-\hat{A}_{2, \mathrm{H}} \hat{B}_{1, \mathrm{H}}\right) \mathbf{K} \\
= & h\left(C_{\mathbb{R}}\right)
\end{aligned}
$$

We now discuss the canonical forms of $\mathbb{R}$-linear matrices. Let $A_{\mathbb{R}}$ be an $\mathbb{R}$-linear transformation, $\hat{A}_{\mathbb{R}}$ its real counterpart, $\left\{\lambda_{1}+i \mu_{1}, \lambda_{2}+i \mu_{2}, \ldots, \lambda_{s}+i \mu_{s}\right\}$ the complex eigenvalues of $\hat{A}_{\mathbb{R}}$, and $\left\{\lambda_{2 s+1}, \ldots, \lambda_{4 n}\right\}$ the real eigenvalues of $\hat{A}_{\mathbb{R}}$. As well known [19,20], there exists a real orthogonal matrix $O$ such that

$$
\hat{J}_{\mathbb{R}}=O \hat{A}_{\mathbb{R}} O^{T},
$$

where

$$
\begin{aligned}
& \hat{J}_{\mathbb{R}}=\left(\begin{array}{ccccccc}
X_{1} & & & & & & \\
& X_{2} & & & & & P \\
& & \ddots & & & & \\
& & & X_{s} & & & \\
& 0 & & & \lambda_{2 s+1} & & \\
& & & & & \ddots & \\
& & & & & & \lambda_{4 n}
\end{array}\right) \\
& =\left(\begin{array}{ccccccc}
X_{1} & & & & & & \\
& X_{2} & & & & 0 & \\
& & \ddots & & & & \\
& & & X_{s} & & & \\
& 0 & & & \lambda_{2 s+1} & & \\
& & & & & \ddots & \\
& & & & & & \lambda_{4 n}
\end{array}\right)+\left(\begin{array}{cccccc}
0 & & & & \\
& 0 & & & P & \\
& & \ddots & & & \\
& & 0 & & \\
& 0 & & 0 & \\
& & & & \ddots & \\
& & & & & 0
\end{array}\right) \\
& =\hat{D}_{\mathbb{R}}+\hat{N}_{\mathbb{R}} .
\end{aligned}
$$

In the previous equation, $X_{r}$ represents a $2 \times 2$ real matrix with eigenvalues $\lambda_{r} \pm i \mu_{r}$. An appropriate choice of $O$ guarantees that

$$
X_{r}=\left(\begin{array}{cc}
\lambda_{r} & -\mu_{r} \\
\mu_{r} & \lambda_{r}
\end{array}\right) .
$$

Let us come back to the $\mathbb{R}$-linear transformation $A_{\mathbb{R}}$. By using the translation rules given in Eqs. (24,25), we can immediately give its canonical form

$$
J_{\mathbb{R}}=D_{\mathbb{R}}+N_{\mathbb{R}} .
$$

In particular, the diagonal elements of $D_{\mathbb{R}}$ corresponding to the quaternionic translation of the real blocks

$$
\begin{aligned}
\hat{D}_{1, \mathbb{R}} & =\left(\begin{array}{cccc}
\lambda_{m} & -\mu_{m} & 0 & 0 \\
\mu_{m} & \lambda_{m} & 0 & 0 \\
0 & 0 & \lambda_{m+1} & -\mu_{m+1} \\
0 & 0 & \mu_{m+1} & \lambda_{m+1}
\end{array}\right), \\
\hat{D}_{2, \mathbb{R}} & =\left(\begin{array}{cccc}
\lambda_{m} & -\mu_{m} & 0 & 0 \\
\mu_{m} & \lambda_{m} & 0 & 0 \\
0 & 0 & \lambda_{m+1} & 0 \\
0 & 0 & 0 & \lambda_{m+2}
\end{array}\right), \\
\hat{D}_{3, \mathbb{R}} & =\left(\begin{array}{cccc}
\lambda_{m} & 0 & 0 & 0 \\
0 & \lambda_{m+1} & 0 & 0 \\
0 & 0 & \lambda_{m+2} & 0 \\
0 & 0 & 0 & \lambda_{m+3}
\end{array}\right)
\end{aligned}
$$


are respectively given by

$$
\begin{aligned}
D_{1, \mathbb{R}}= & \frac{1}{2}[ \\
\quad & \lambda_{m}\left(1-L_{i} R_{i}\right)+\mu_{m}\left(L_{i}+R_{i}\right)+ \\
D_{2, \mathbb{R}}=\frac{1}{2}[ & \lambda_{m}\left(1-L_{i} R_{i}\right)+\mu_{m}\left(L_{i}+R_{i}\right)+ \\
& \left.\frac{1}{2} \lambda_{m+1}\left(1+L_{i} R_{i}-L_{j} R_{j}+L_{k} R_{k}\right)+\frac{1}{2} \lambda_{m+2}\left(1+L_{i} R_{i}+L_{j} R_{j}-L_{k} R_{k}\right)\right], \\
D_{3, \mathbb{R}}=\frac{1}{4}[ & \lambda_{m}\left(1-L_{i} R_{i}-L_{j} R_{j}-L_{k} R_{k}\right)+\lambda_{m+1}\left(1-L_{i} R_{i}+L_{j} R_{j}+L_{k} R_{k}\right)+ \\
& \left.\lambda_{m+2}\left(1+L_{i} R_{i}-L_{j} R_{j}+L_{k} R_{k}\right)+\lambda_{m+3}\left(1+L_{i} R_{i}+L_{j} R_{j}-L_{k} R_{k}\right)\right] .
\end{aligned}
$$

As happens for $\mathbb{C}$-linear quaternionic matrices, an $\mathbb{R}$-linear quaternionic matrix is diagonalizable if the corresponding real counterpart is diagonalizable. The converse is not necessarily true.

\section{THE EIGENVALUE PROBLEM FOR $\mathbb{R}$-LINEAR MATRICES}

Let us now consider the eigenvalue problem for $\mathbb{R}$-linear quaternionic matrices. Eq. (13) is obviously too restrictive. In fact, such an equation sets the real eigenvalue spectrum of $\mathbb{R}$-linear quaternionic operators. No information is given about the remaining eigenvalues. In particular, if the real counterpart $\hat{M}_{\mathbb{R}}$ of the $\mathbb{R}$-linear quaternionic matrix $M_{\mathbb{R}}$ does not have real eigenvalues, Eq. (13) does not admit solution. This is very embarrassing if we consider, for example, $\mathbb{R}$-linear anti-hermitian quaternionic operators. Thus, we need to modify Eq. (13). The discussion regarding the "pseudo-triangular"

form of the matrices $\hat{A}_{\mathbb{R}}$ [see Eq. (26)] suggests as $\mathbb{R}$-linear eigenvalue problem the following system of coupled equations

$$
\begin{aligned}
& M_{\mathbb{R}} \psi=a \psi+b \varphi \\
& M_{\mathbb{R}} \varphi=c \varphi+d \psi
\end{aligned}
$$

where

$$
a, b, c, d \in \mathbb{R}, \quad \psi=\psi_{0}+i \psi_{1}+j \psi_{2}+k \psi_{3}, \quad \varphi=\varphi_{0}+i \varphi_{1}+j \varphi_{2}+k \varphi_{3}, \quad \psi_{0, \ldots, 3}, \varphi_{0, \ldots, 3} \in V[n, \mathbb{R}] .
$$

It can be shown that the real coefficients $a, b, c, d$ are related to the real and imaginary part of the $\hat{M}_{\mathbb{R}}$ eigenvalues. In fact, by translating the system (30) into its real matrix counterpart, we find

$$
\left(\begin{array}{cc}
\hat{M}_{\mathbb{R}} & 0 \\
0 & \hat{M}_{\mathbb{R}}
\end{array}\right)\left(\begin{array}{c}
\hat{\psi} \\
\hat{\varphi}
\end{array}\right)=\left(\begin{array}{ll}
a \mathbf{1}_{4 n} & b \mathbf{1}_{4 n} \\
c \mathbf{1}_{4 n} & d \mathbf{1}_{4 n}
\end{array}\right)\left(\begin{array}{c}
\hat{\psi} \\
\hat{\varphi}
\end{array}\right)
$$

where

$$
\hat{\psi}=\left(\begin{array}{c}
\psi_{0} \\
\psi_{1} \\
\psi_{2} \\
\psi_{3}
\end{array}\right), \quad \hat{\varphi}=\left(\begin{array}{c}
\varphi_{0} \\
\varphi_{1} \\
\varphi_{2} \\
\varphi_{3}
\end{array}\right) \quad \in V[4 n, \mathbb{R}]
$$

The matrix equation (31) admits non trivial solutions if and only if

$$
\operatorname{det}\left[\left(\begin{array}{cc}
\hat{M}_{\mathbb{R}}-a \mathbf{1}_{4 n} & -b \mathbf{1}_{4 n} \\
-c \mathbf{1}_{4 n} & \hat{M}_{\mathbb{R}}-d \mathbf{1}_{4 n}
\end{array}\right)\right]=0 .
$$

By rewriting the matrix $\hat{M}_{\mathbb{R}}$ in terms of the symilarity matrix $\hat{S}_{\mathbb{R}}$ and of its Jordan form $\hat{J}_{\mathbb{R}}$, i.e.

$$
\hat{M}_{\mathbb{R}}=\hat{S}_{\mathbb{R}} \hat{J}_{\mathbb{R}} \hat{S}_{\mathbb{R}}^{-1}
$$

and by using the cyclic property of the determinant, we reduce Eq.(32) to

$$
\operatorname{det}\left[\left(\begin{array}{cc}
\hat{J}_{\mathbb{R}}-a \mathbf{1}_{4 n} & -b \mathbf{1}_{4 n} \\
-c \mathbf{1}_{4 n} & \hat{J}_{\mathbb{R}}-d \mathbf{1}_{4 n}
\end{array}\right)\right]=0 .
$$


By simple algebraic manipulations [20], we obtain

$$
\begin{aligned}
\operatorname{det}\left[\left(\begin{array}{cc}
\hat{J}_{\mathbb{R}}-a \mathbf{1}_{4 n} & -b \mathbf{1}_{4 n} \\
-c \mathbf{1}_{4 n} & \hat{J}_{\mathbb{R}}-d \mathbf{1}_{4 n}
\end{array}\right)\right] & =\operatorname{det}\left[\left(\hat{J}_{\mathbb{R}}-a \mathbf{1}_{4 n}\right)\left(\hat{J}_{\mathbb{R}}-d \mathbf{1}_{4 n}\right)-b c \mathbf{1}_{4 n}\right] \\
& =\operatorname{det}\left[\hat{J}_{\mathbb{R}}^{2}-(a+d) \hat{J}_{\mathbb{R}}+(a d-b c) \mathbf{1}_{4 n}\right] \\
& =\prod_{i}\left[z_{i}^{2}-(a+d) z_{i}+(a d-b c)\right] \\
& =0
\end{aligned}
$$

where $z_{i}$ represent the eigenvalues of the real matrix $\hat{M}_{\mathbb{R}}$. The previous equation explicitly shows the relation between the real coefficients $a, b, c, d$ (which appear in the $\mathbb{R}$-linear eigenvalue problem) and the eigenvalues $z$ of the real counterpart of the quaternionic matrix $M_{\mathbb{R}}$. In the case of complex eigenvalues $z$, we find

$$
\Delta=(a+d)-4(a d-b c)=(a-d)^{2}+b c<0 .
$$

This condition guarantees that the eigenvalues of the real matrix

$$
Z=\left(\begin{array}{ll}
a \mathbf{1}_{4 n} & b \mathbf{1}_{4 n} \\
c \mathbf{1}_{4 n} & d \mathbf{1}_{4 n}
\end{array}\right)
$$

appear in conjugate pairs. Consequently, we can find a real similarity transformation $T$ such that

$$
T Z T^{-1}=\left(\begin{array}{cc}
\lambda \mathbf{1}_{4 n} & -\mu \mathbf{1}_{4 n} \\
\mu \mathbf{1}_{4 n} & \lambda \mathbf{1}_{4 n}
\end{array}\right) .
$$

Finally, without loss of generality, we can consider the following eigenvalue problem for $\mathbb{R}$-linear transformations

$$
\begin{aligned}
& M_{\mathbb{R}} \psi=\lambda \psi-\mu \varphi, \\
& M_{\mathbb{R}} \varphi=\lambda \varphi+\mu \psi .
\end{aligned}
$$

\section{FINAL REMARKS}

These final remarks aim to give a concluding discussion on the "coupled" eigenvalue problem and a brief summary of mathematical and physical applications motivating our interest in this research. In particular, we are interested to bring together two areas: quaternionic differential operators and quantum mechanics.

\section{Coupled eigenvalue equations}

In the previous Section, we have introduced, for $\mathbb{R}$-linear transformations, the eigenvalue problem (35) which represents the natural generalization of (13). In particular, as we observed above, the study of system (35) instead of Eq.(13) allows to take into account the existence of complex eigenvalues and, consequently, complete the eigenvalue spectrum of $\mathbb{R}$-linear quaternionic operators. Actually, the eigenvalue problem (35) also applies to $\mathbb{H}$ and $\mathbb{C}$-linear transformations. It can be considered as an equivalent formulation of Eqs.(9) and (12). To show that, let us consider the equation

$$
M_{\mathrm{C}} \psi=\psi z=\psi \lambda+\psi i \mu .
$$

We limit ourselves to discuss $\mathbb{C}$-linear transformations. Obviously, if a preferred complex direction is chosen for the eigenvalues of $\mathbb{H}$-linear quaternionic operators, all the arguments in what follows also hold for $\mathbb{H}$-linear transformations. By using the $\mathbb{C}$-linearity, we find

$$
\left(M_{\mathbb{C}} \psi\right) i=M_{\mathbb{C}}(\psi i)=\psi i \lambda-\psi \mu .
$$

The pair of eigenvector $(\psi, \varphi=\psi i)$, where $\psi$ is solution of Eq.(36), satisfies the coupled equations

$$
\begin{aligned}
& M_{\mathbb{C}} \psi=\lambda \psi-\mu \varphi, \\
& M_{\mathbb{C}} \varphi=\mu \psi+\lambda \varphi .
\end{aligned}
$$


Vice-versa, let $M_{\mathbb{C}}$ and $(37)$ be respectively a $\mathbb{C}$-linear transformation and the corresponding eigenvalue problem. We denote by $(\psi, \varphi)$ a solution of the system (37). If $\psi$ satisfies Eq.(36) too, then, comparing Eq.(36) and the first equation in (37), one immediately obtains $\varphi=\psi i$. If, on the contrary, $\psi$ is not a solution of Eq.(36), by using the $\mathbb{C}$-linearity, we obtain

$$
M_{\mathbb{C}}(\varphi i)=\left(M_{\mathbb{C}} \varphi\right) i=\mu \psi i+\varphi i
$$

Thus,

$$
M_{\mathbb{C}}(\psi+\varphi i)=(\psi+\varphi i) z .
$$

Hence, it is possible to associate to any solution $(\psi, \varphi)$ of the system (37) a corresponding eigenvector of $M_{\mathbb{C}}$.

It is worth pointing out that the coupled system (35) can be obtained by solving the eigenvalue problem (13) for complexified quaternionic eigenvectors and complexified real eigenvalues. Infact, by imposing that

$$
\psi \rightarrow \Psi=\psi+I \varphi \quad \in \mathbb{H}(1, i, j, k) \otimes \mathbb{C}(1, I),
$$

and

$$
r \rightarrow Z=\lambda+I \mu \quad \in \mathbb{C}(1, I),
$$

from the complexified eigenvalue problem (13),

$$
M_{\mathbb{R}} \Psi=\Psi Z,
$$

we immediately get the coupled system $(35)$.

\section{Applications}

Many physical problems dealing with differential operators are greatly simplified by using the matrix formalism and solving the corresponding eigenvalue problem.

Let us first consider a very simple case. That is the $\mathbb{H}$-linear second order homogeneous ordinary differential equation

$$
\ddot{\psi}(x)-\alpha \dot{\psi}(x)-\beta \psi(x)=0, \quad \alpha, \beta \in \mathbb{H}, x \in \mathbb{R} .
$$

In looking for quaternionic exponential solution $\psi(x)=\exp [q x]$ and observing that the derivative of $\exp [q x]$ with respect to the real variable $x$ is $q \exp [q x]$, we reduce the previous problem to find the solutions of the following quadratic equation

$$
q^{2}=\alpha q+\beta .
$$

This equation can be rewritten in matrix form as follows

$$
M_{\mathbb{H}}\left(\begin{array}{c}
q \\
1
\end{array}\right)=\left(\begin{array}{c}
q^{2} \\
q
\end{array}\right), \quad M_{\mathbb{H}}=\left(\begin{array}{cc}
\alpha & \beta \\
1 & 0
\end{array}\right) .
$$

As seen in this paper, the $\mathbb{H}$-linear quaternionic matrix $M_{\mathbb{H}}$ satisfies a right (complex) eigenvalue equation

$$
M_{\mathbb{H}}\left(\begin{array}{c}
v \\
w
\end{array}\right)=\left(\begin{array}{c}
v \\
w
\end{array}\right) z, \quad z \in \mathbb{C}, v, w \in \mathbb{H}, w \bar{w}=1 .
$$

Due to the particular form of $M_{\mathbb{H}}$, the components of the $M_{\mathbb{H}}$-eigenvectors satisfy the following condition

$$
v=w z .
$$

Multiplying (from the right) Eq. (42) by $\bar{w}$ and using the constraint (43), we obtain

$$
M_{\mathbb{H}}\left(\begin{array}{c}
w z \bar{w} \\
1
\end{array}\right)=\left(\begin{array}{c}
w z^{2} \bar{w} \\
w z \bar{w}
\end{array}\right) .
$$


Comparing Eq. (41) with Eq. (44), we immediately get

$$
q=w z \bar{w} .
$$

The problem of finding exponential solutions for $\mathbb{H}$-linear differential with constant coefficients and, consequently, zeros of $\mathbb{H}$-linear polynomial equations [21], is thus equivalent to solve the right (complex) eigenvalue problem for the associated matrix. Obviously, the previous considerations also hold for the $n$-dimensional case.

The solutions of $\mathbb{X}$-linear quaternionic differential equations with constant coefficients

$$
\psi^{(n)}(x)-A_{n-1, \mathbb{x}} \psi^{(n-1)}(x)-A_{n-2, \mathrm{x}} \psi^{(n-2)}(x)-\ldots-A_{0, \mathrm{x}} \psi(x)=0, \quad \mathbb{X}=\mathbb{R}, \mathbb{C}, \mathbb{H} .
$$

can be given in terms of the eigenvalues and eigenvectors of the matrix

$$
\left(\begin{array}{cccc}
A_{n-1, \mathrm{x}} & A_{n-2, \mathrm{x}} & \ldots & A_{0, \mathrm{x}} \\
1 & 0 & \ldots & 0 \\
\cdot & \cdot & \ldots & \cdot \\
\cdot & \cdot & \cdots & \cdot \\
\cdot & \cdot & \ldots & \cdot \\
0 & 0 & \ldots & 0
\end{array}\right)
$$

Interesting $\mathbb{C}$-linear differential equations appear in quaternionic quantum mechanics [3]. For example, by studying quaternionic tunneling effect as candidate to possible phenomenological deviations from the standard (complex) theory, we have to solve the following $\mathbb{H}$-linear Schrödinger equation

$$
\partial_{t} \Psi(x, t)=\left[\frac{i}{\hbar}\left(\frac{\hbar^{2}}{2 m} \partial_{x x}-i V\right)+\frac{j}{\hbar} W\right] \Psi(x, t)
$$

where $\frac{j}{\hbar} W$ represents the new quaternionic perturbation. The quaternionic stationary state wave function

$$
\Psi(x, t)=\psi(x) \exp \left[-\frac{i}{\hbar} E t\right]
$$

is solution of Eq. (47) on the condition that $\psi(x)$ be solution of the following time-independent $\mathbb{C}$-linear (ordinary) differential equation

$$
i \frac{\hbar^{2}}{2 m} \ddot{\psi}(x)-i V \psi(x)+j W \psi(x)+\psi(x) i E=0 .
$$

Observe that the choice of the imaginary unit $i$ in the Laplacian operator $\partial_{x x}$, Eq.(47), and in the time exponential, Eq.(48), is fundamental to recover the standard results in the complex limit. In this formalism, quaternionic potentials are treated as perturbation effects on standard quantum mechanics. We also point out that the right position of the time exponential is fundamental to perform the separation of variables.

The solution of Eq.(49) can be given in terms of the eigenvalues and eigenvectors of the $\mathbb{C}$-linear matrix

$$
\left(\begin{array}{cc}
0 & A_{0, \mathbb{c}} \\
1 & 0
\end{array}\right)
$$

where

$$
A_{0, \mathrm{C}}=\frac{2 m}{\hbar^{2}}\left(V+L_{k} W+L_{i} R_{i} E\right) .
$$

A detailed phenomenological discussion of the quaternionic tunneling effect is found in the paper of ref. 近.

\section{Outlooks}

As seen in this paper, the choice of right (complex) eigenvalues for $\mathbb{H}$ and $\mathbb{C}$ linear operators play a fundamental role in discussing canonical forms, in finding solutions of polynomial and differential equations. It was shown that the right (complex) eigenvalue problem is equivalent to a "coupled" system and this was extremely important to study the eigenvalue problem for $\mathbb{R}$-linear quaternionic matrices, where a pair of real eigenvalues must be introduced. This work was intended as an attempt 
at motivating the study of $\mathbb{R}$ and $\mathbb{C}$-linear quaternionic operators in view of possible applications in quantum mechanics and gauge theory. It would be desirable to give a complete theory of $\mathbb{X}$-linear quaternionic matrices and differential operators. More realistically, this paper touches only a few aspects of the theory and shows how the choice of the right eigenvalue equation seems to be the best to investigate quaternionic formulations of physical theory. It was not our purpose to study here differential operators. The results in this field are far from being conclusive and some questions represent at present intriguing challenges: variations of parameters; order reduction; not invertible higher derivative $\mathbb{R}$ and $\mathbb{C}$-linear constant coefficients; variable coefficients; integral transforms. Finally, it would be desirable to extend the discussion on the eigenvalue problem by matrix translation to the non-associative case [22, 23, 24]

\section{Acknowledgments}

The authors wish to express their thanks to Profs. N. Cohen and G. Ducati for the helpful discussions during the preparation of the paper and for drawing their attention to some interesting applications in matrix and differential operator theory. The authors also thank an anonymous referee for comments, references and suggestions. One of the authors (SDL) gratefully acknowledges the University of Lecce (Department of Physics) and Curitiba (Department of Mathematics) for the hospitality, and the FAEP (State University of Campinas) for financial support.

\section{References}

1. S. De Leo and G. Scolarici, "Right eigenvalue equation in quaternionic quantum mechanics", J. Phys. A 33, 2971-2995 (2000).

2. S. De Leo and G. Ducati, "Quaternionic differential operators", J. Math. Phys. 42, 2236-2265 (2001).

3. S. Adler, Quaternionic quantum mechanics and quantum fields, (Oxford University Press, New York, 1995).

4. S. De Leo, G. Ducati and C. Nishi, "Violations of Quantum Mechanics" hep-th/0111143, to appear in J. Phys. A].

5. S. De Leo, "Quaternionic factorizations of Dirac's equation" [in preparation].

6. L. P. Horwitz and L. C. Biedenharn, "Quaternion Quantum Mechanics: Second Quantization and Gauge Fields", Ann. Phys. 157, 432-488 (1984).

7. L. Huang, "On two questions about quaternion matrices", Lin. Alg. Appl. 318, 79-86 (2000).

8. L. Huang and W. So, "On left eigenvalues of a quaternionic matrix", Lin. Alg. Appl. 323, 105-116 (2001).

9. T. Dray and C. Manogue, The octonionic eigenvalue problem, Adv. Cliff. Alg. 8, 341-364 (1998).

10. F. Zhang, "Quaternions and Matrices of Quaternions", Lin. Alg. Appl. 251, 21-57 (1997).

11. Y. H. A. Yueng, "Another proof of the theorems on the eigenvalues of a square quaternion matrix", Proc. Glashow Math. Assoc. 6, 191-195 (1964).

12. R. M. Wood, "Quaternionic eigenvalues", Bull. London Math. Soc. 17, 137-138 (1985).

13. P. M. Cohn, Skew field constructions, (Cambridge University Press, New York, 1977).

14. J. L. Brenner, "Matrices of quaternions", Pacific J. Math. 1, 329-335 (1951).

15. H. C. Lee, "Eigenvalues and canonical forms of matrices with quaternion coefficients", Proc. R. Irish. Acad. A 52, 253-260 (1949).

16. N. A. Wiegmann, "Some theorems on matrices with real quaternion elements", Canad. J. Math. 7 191-201 (1955).

17. P. Rotelli, "The Dirac equation on the quaternionic field", Mod. Phys. Lett. A 4, 933-940 (1989).

18. S. De Leo, "Quaternionic Lorentz group and Dirac equation", Found. Phys. Lett. 14, 37-50 (2001).

19. J. H. Wilkinson, The Algebraic Eigenvalue Problem (Oxford University Press, New York, 1965).

20. S. Barnet, Matrices Methods and Applications, (Oxford University Press, New York, 1990).

21. R. Serôdio and Lok-Shun Siu, Zeros of quaternion polynomials, Appl. Math. Lett. 14, 237-239 (2001).

22. S. De Leo and K. Abdel-Khalek, Ocotnionic representations of $G L(8, R)$ and $G L(4, C)$, J. Math. Phys. 38, 582-598 (1997).

23. T. Dray and C. Manogue, Finding octonionic eigenvectors using Mathematica, Comp. Phys. Commun. 115, 536-547 (1998).

24. T. Dray and C. Manogue, The exceptional Jordan eigenvalue problem, Int. J. Theor. Phys. 38, 2197-2220 (1999). 